\title{
La révolution de 1848 dans les « comédies politiques » de Johann Nestroy
}

The Revolution of 1848 in the "political comedies" of Johann Nestroy

\section{Fanny Platelle}

\section{(2) OpenEdition \\ 1 Journals}

\section{Édition électronique}

URL : https://journals.openedition.org/ahrf/12440

DOI : $10.4000 /$ ahrf. 12440

ISSN : 1952-403X

Éditeur :

Armand Colin, Société des études robespierristes

\section{Édition imprimée}

Date de publication : 1 mars 2012

Pagination : 145-172

ISSN : 0003-4436

\section{Référence électronique}

Fanny Platelle, «La révolution de 1848 dans les « comédies politiques » de Johann Nestroy », Annales historiques de la Révolution française [En ligne], 367 | janvier-mars 2012, mis en ligne le 28 septembre 2012, consulté le 01 juillet 2021. URL : http://journals.openedition.org/ahrf/12440 ; DOI : https:// doi.org/10.4000/ahrf.12440 


\title{
LA RÉVOLUTION DE 1848 \\ DANS LES « COMÉDIES POLITIQUES » DE JOHANN NESTROY
}

Fanny PLATELLE

\begin{abstract}
« Et je hochais la tête, et c'était tout, ce que je pouvais faire dans ces circonstances particulières, Et je le faisais de manière significative $»{ }^{1}$.
\end{abstract}

\begin{abstract}
Les « comédies politiques » de Johann Nestroy, écrites en 1848-50, représentent la Révolution viennoise de 1848 , sa répression et les conséquences de la réaction. Loin de manifester un " changement de cap » de l'auteur après 1848 , elles révèlent la continuité de sa position : tout en saluant les acquis révolutionnaires, Nestroy doute du succès de la Révolution et critique par la satire et la parodie les faiblesses politiques et « humaines » des deux camps, qui empêchent la réalisation des idéaux. Par son intention critique et didactique, il annonce la « comédie politique » de B. Brecht ou de F. Dürrenmatt.

Mots-clés : Johann Nestroy, théâtre populaire viennois, Révolution de 1848, comédies politiques, satire, parodie
\end{abstract}

L'œuvre dramatique de Johann Nestroy (1801-1862) s'étend de 1827 (Der Zettelträger Papp) à 1862 (Der Häuptling Abendwind oder Das greuliche Festmahl) et comprend donc la période de la Révolution de 1848 , qui débute à Vienne le 13 mars et s'achève par la capitulation

(1) Aphorisme des œuvres posthumes, cité d'après Johann Nestroy. Freiheit in Krähwinkel, Jürgen HeIn (ed.), Stuttgart, Reclam [UB 8330], 1998, p. 87. 
de la ville le $1^{\text {er }}$ novembre ${ }^{2}$. Cet événement a signifié pendant plusieurs mois pour l'auteur la liberté d'écrire et de jouer, la censure étant abolie le 14 mars 1848 et rétablie le 11 novembre 1848 . Les « comédies politiques $»^{3}$, écrites en 1848-50, représentent la Révolution et la réaction qui suivit: Freiheit in Krähwinkel (Liberté à Krähwinkel, première le

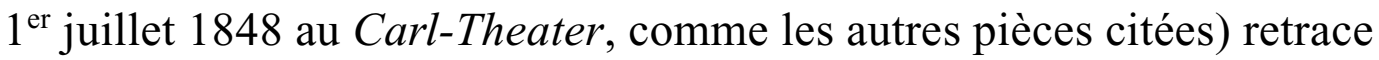
le printemps révolutionnaire, Lady und Schneider (Lady et tailleur, première le 6 février 1849) dresse dans les premiers mois de la réaction un bilan désabusé de la Révolution, Judith und Holofernes (Judith et Holopherne, parodie de Judith de F. Hebbel, première le 13 mars 1849) évoque le siège de Vienne en octobre 1848, Höllenangst (Peur de l'Enfer ou Peur bleue, première le 17 novembre 1849) et Der alte Mann mit der jungen Frau (Le vieil homme avec la jeune femme, écrite en 1849, mais non représentée du vivant de Nestroy à cause de la censure ${ }^{4}$ ) montrent les conséquences de la réaction. Des allusions à la Révolution sont également présentes dans les autres pièces de la période, en particulier Die lieben Anverwandten ${ }^{5}$ (Les chers parents, première le 21 mai 1848) et Verwickelte Geschichte! (Histoire embrouillée! première le 22 juin $1850)^{6}$. Les farces ont suscité dès l'époque des commentaires opposés : les critiques louent la représentation fidèle des événements révolutionnaires dans Freiheit in Krähwinkel ${ }^{7}$ ou reprochent au contraire à l'auteur d'avoir moqué l'aspiration des Viennois à la liberté ${ }^{8}$. Et la position poli-

(2) Cf. Heinrich Reschauer, Moritz Smets, Das Jahr 1848. Geschichte der Wiener Revolution, 2 vol., Wien, Waldheim, 1872.

(3) Selon le titre du tome 5 de Johann Nestroy. Sämtliche Werke. Historisch-kritische Gesamtausgabe in zwölf Bändern, Fritz BRUKNER et Otto Rommel (ed.) avec la collaboration de Adolf Hoffmann, t. V : Politische Komödien, Wien, Verlag Anton Schroll \& Co, 1925. [Cité SW V]

(4) Dans cette farce (comme dans Freiheit in Krähwinkel), les opinions politiques de Nestroy sont intégrées à la trame de l'action et intimement liées au caractère du protagoniste Kern, ce qui rendait tout remaniement impossible.

(5) Nestroy a vraisemblablement achevé l'adaptation du roman de Charles Dickens, Martin Chuzzlewit, en 1847, et ajouté avant la première le commentaire des événements contemporains dans les « couplets » et monologues.

(6) Voir aussi Sie sollen ihn nicht haben oder Der holländische Bauer et KarikaturenCharivari mit Heiratszweck (1850).

(7) Ainsi le Österreichischer Courier du 3 juillet 1848, in SW V, p. 630; ou le tract anonyme (1848) : « Nestroy und die Freiheit in Krähwinkel oder was hat Nestroy für einen Karakter, und was haben wir von ihm zu hoffen? », dans Johann Nestroy. Gesammelte Briefe und Revolutionsdokumente, Fritz BruKnER (ed.), Wien, Wallishausser, 1938, p. 113 sq.

(8) Der Humorist du 3 juillet 1848, ibid., p. 112 sq. Karl Kraus qualifie quant à lui la pièce de « satire antirévolutionnaire », " Nestroy und die Nachwelt» dans Die Fackel (1912), cité d'après Werke, Heinrich FISCHER (ed.), t. 8, München, 1960, p. 233 sq. 
tique de Nestroy ${ }^{9}$ est l'objet de débats jusqu'à nos jours, certains considérant l'auteur comme « un libéral, un ami du peuple, un démocrate $»^{10}$, d'autres le décrivant comme un "génie de l'opportunisme », qui choisit toujours « la solution de facilité $»^{11}$.

Nestroy est-il un simple "chroniqueur 》 des événements révolutionnaires? Comment la Révolution de 1848 est-elle dépeinte dans ses farces : quelle image en donne-t-il, quelle position adopte-t-il et quelle est son intention? Afin de répondre à ces questions, nous étudierons dans un premier temps la représentation de la Révolution et de ses conséquences dans les comédies politiques, en montrant que l'auteur pressent, dès le printemps 1848 , le triomphe de la réaction, puis nous analyserons les raisons de l'échec du mouvement révolutionnaire mises en évidence dans les pièces.

\section{La représentation de la Révolution et de ses conséquences}

\section{Les comédies politiques comme miroir des événements de 1848-49}

Freiheit in Krähwinkel est la seule farce de Nestroy entièrement consacrée à la Révolution. L'auteur reprend, pour relater les événements du printemps 1848, le motif de Krähwinkel. Krähwinkel est le « nom d'un lieu fictif, modèle de la petite ville étriquée $\gg^{12}$. Jean Paul est le premier à l'utiliser avec une intention satirique dans Das heimliche Klagelied der jetzigen Männer : eine Stadtgeschichte; - und die wunderbare Gesellschaft in der Neujahrsnacht (1801). Un an plus tard, August von Kotzebue fait représenter la comédie Die deutschen Kleinstädter (1802), inspirée de la Petite ville de Picard (1797), puis il réutilise le motif de Krähwinkel avec une fonction satirique dans deux suites (en 1806-1809 et 1810). Le théâtre populaire viennois hérite de ce topos burlesque : jusqu'en 1832, une quinzaine de pièces de dramaturges viennois (Castelli, Meisl, Gleich, Bäuerle etc.) ont pour motif central Krähwinkel, dont la plus célèbre, Die

(9) Du point de vue biographique, la participation de Nestroy à la Révolution de 1848 dans la garde nationale avec son collègue Wenzel Scholz relève davantage de la publicité pour le Carltheater que d'un véritable engagement. Cf. Johann Nestroy. Historisch-kritische Ausgabe. Stücke 26/I Freiheit in Krähwinkel, John R.P. McKenzIE (ed.), Wien, Jugend und Volk, 1995, p. 94-95. [Noté HKA dans la suite de l'article]

(10) Tract anonyme cité note 7.

(11) Otto Basil, J. Nestroy in Selbstzeugnissen und Bilddokumenten, Reinbek, Rowohlt, 1966, p. 126.

(12) Jacob et Wilhelm Grimm, Deutsches Wörterbuch, Leipzig, Hirzel, 1873, t. 5, colonne 1975 sq. 
falsche Primadonna in Krähwinkel d'Adolf Bäuerle (1818), est la source de Nestroy.

L'auteur, qui emprunte à la tradition les principaux personnages et la trame dramatique de sa farce, donne au motif, devenu cliché, une fonction nouvelle, critique de l'époque : Krähwinkel ne renvoie pas à n'importe quelle petite ville, mais à Vienne et à l'Autriche. Krähwinkel est « Vienne codée $»^{13}$, la satire vise un lieu précis et les événements contemporains. Les personnages deviennent les partisans et opposants de la Révolution. Le porte-parole de Nestroy, Ultra, souligne la fonction de «modèle » de Krähwinkel :

«Tous les éléments d'une révolution, tout ce qui révolte l'humanité, qu'ils ont ailleurs en grand, nous l'avons, habitants de Krähwinkel, en petit. Nous avons un petit tyran absolu, nous avons un petit gouvernement irresponsable, une petite bureaucratie, une petite censure, une petite dette publique, qui dépasse largement nos petites forces, alors nous devons avoir aussi une petite révolution et grâce à cette petite révolution une petite constitution et enfin une petite liberté $\rangle^{14}$.

Krähwinkel permet à l'auteur de représenter de manière satirique les événements qui se déroulent dans la réalité contemporaine.

Freiheit in Krähwinkel dépeint les événements qui se déroulèrent à Vienne entre le 13 mars et le 27 mai 1848 (date à laquelle le gouvernement cède après la construction des barricades). Outre l'aspiration générale des habitants à la liberté (chœur I, 1), la farce fait référence à des événements précis ${ }^{15}$ : les trois rêves du maire montrent le début de la Révolution (I, 21), la défense victorieuse des acquis révolutionnaires (I, 23 : la Sturmpetition) et l'espoir des réactionnaires autrichiens en une intervention russe pour réprimer l'insurrection (I, 25). Ce dernier rêve anticipe le rôle des troupes russes lors de la répression de la Révolution hongroise (août 1849). Sont également évoqués la démission de Metternich le 13 mars et son exil à Londres ${ }^{16}$ (III, 13, 15, 24), l'abolition de la censure le 14 mars $(I, 8)$, la proclamation de la constitution au

(13) Freiheit in Krähwinkel, Reclam, p. 82.

(14) HKA 26/I, I, 8, p. 21. Voir aussi les scènes I, 7 et III, 25. Les traductions sont faites par nous.

(15) Les principaux événements de la période révolutionnaire sont rappelés dans les autres pièces (en particulier dans les chants et monologues de Höllenangst et Lady und Schneider).

(16) Dans Die lieben Anverwandten, il est décrit comme un « fantôme », qui erre " en plein jour, dans les rues de Londres ». HKA 25/II Die lieben Anverwandten, Friedrich WALLA (ed.), Wien, Deuticke, 1995, I, 5, p. 12. 
peuple par le dramaturge Friedrich Kaiser à cheval le 15 mars (II, 16), l'expulsion des Liguoriens le 6 avril (III, 5-12) et la construction des barricades le 26 mai (III, 17 et III, 22).

La farce fait en outre allusion à des institutions et aspects caractéristiques de l'époque : aux différentes légions (à la garde nationale ${ }^{17}-\mathrm{I}$, 1 - et à la légion académique - III, 24), au Haslinger, bâton de noisetier dont étaient armés les policiers et symbole de la peine de la bastonnade, abolie en mars 1848 (I, 9) ${ }^{18}$, au charivari que faisaient les révolutionnaires devant le domicile de certaines personnes afin de manifester leur mécontentement $(\mathrm{I}, 10)^{19}$, au rôle de la presse locale et étrangère (I, 3; I, 8), aux drapeaux nationaux (de la monarchie - I, 24 - et de la Révolution - II, 15), à la poésie de circonstance (II, 2-3; III, 1), au costume des révolutionnaires (le chapeau Kalabreser - III, 24).

Quant aux protagonistes, ils évoquent des personnages historiques : le maire de Krähwinkel rappelle Ignaz Czapka, maire de Vienne de 1838 à 1848 (I, 9; III, 2), le tyran militaire Rummelpuff le maréchal Windischgrätz (II, 7), le fonctionnaire municipal Klaus Josef Sedlnitzky, chef de la police et de la censure de Vienne de 1817 à 1848 (I, 6), de même les Liguoriens ${ }^{20}$; ou bien des personnages typiques de l'époque : Ultra se déguise en prince russe armé d'un knout (II, 4-7), puis en ouvrier, dans la première version en prolétaire (III, 19), les jeunes filles de Krähwinkel en étudiants sur les barricades ${ }^{21}$ (III, 21). Enfin, le contexte européen est exposé dans les «couplets $»^{22}$ d'Ultra : le premier (I, 7) retrace les débuts de l'insurrection contre les régimes réactionnaires, qui partit de Paris et se propagea à l'Allemagne et à l'Autriche, tandis que le couplet final (III, 22) annonce l'échec du « gouvernement des prolétaires » pari$\operatorname{siens}^{23}$, la population aspirant au retour d'un souverain.

(17) De même Judith und Holofernes, scènes 11, 17.

(18) Voir aussi Die lieben Anverwandten I, 5 et Verwickelte Geschichte! II, 6 (strophe 4).

(19) Ce rituel collectif, bruyant et dissonant, utilisé par les Viennois en 1848, provient de la pièce de Roderich Benedix, Das bemooste Haupt (première le $1^{\mathrm{er}}$ avril 1848). Cf. Günter Berghaus, J. N. Nestroys Revolutionspossen im Rahmen des Gesamtwerkes. Ein Beitrag zur Bestimmung von Nestroys Weltanschauung auf dem Hintergrund der österreichischen Sozialgeschichte des Vormärz. Thèse, FU Berlin, 1977, p. 100 sq.

(20) La Congrégation du Très Saint Rédempteur avait été instituée à Vienne en 1813 par Klemens Maria Hofbauer; elle comptait en 1848 près de cent prêtres, ecclésiastiques et frères laïcs.

(21) Pour la première fois, les femmes jouaient un rôle actif dans la Révolution.

(22) Le couplet est un chant à refrain qui interrompt l'action et possède un caractère satirique. Voir aussi Höllenangst I, 14.

(23) Allusion au fait que le gouvernement provisoire républicain défendait tout d'abord les intérêts des ouvriers. 
Freiheit in Krähwinkel est une satire de tout ce qui incarne la réaction : l'État absolutiste, l'Église, l'armée et la bureaucratie. Le régime réactionnaire est qualifié par Ultra de «tyrannie » (I, 7-8). Les représen$\operatorname{tants}^{24}$ en sont d'abord le maire, qui affirme : «Le régent est le père, le sujet un petit enfant et la liberté un couteau tranchant » et plus loin : « Je suis le pouvoir et je fais le droit $»^{25}$. Il abhorre la liberté (le charivari est selon lui un « orchestre des Enfers » I, 20). Aussi a-t-il subtilisé la constitution prévue pour Krähwinkel par les grandes puissances européennes (I, 17; II, 4). Il approuve l'usage du knout et est gratifié par Ultra déguisé en prince russe du titre de «Passionski regierski Volkski despotski » (II, 4). Un deuxième représentant est le fonctionnaire municipal Klaus, qui combat avec acharnement tout mouvement en faveur de la liberté. Il déclare lors de son entrée en scène : «Je sers l'État et nous n'aimons pas que l'homme soit libre » et refuse même de sortir à l'air 《libre $»^{26}$. Puisque la pensée présuppose la liberté, Klaus voudrait l'interdire (I, 3). Il qualifie les manifestants d' " armées infernales de la liberté » (I, 19). Un troisième représentant du despotisme est le commandant des soldats de Krähwinkel, le miles gloriosus Rummelpuff, qui dénigre le peuple : «L'homme commence seulement au baron $»^{27}$ ! Les personnages réactionnaires portent généralement un nom ridicule : celui du secrétaire particulier Reakzerl Edler von Zopfen ${ }^{28}$, qui unit deux références à l'ancien régime, révèle son étroitesse d'esprit; l'association de deux noms d'oiseaux pour Sperling Edler von Spatz indique un mauvais poète. En raison de leur bêtise, ces personnages de farce sont dupés par les déguisements d'Ultra. La pièce critique ensuite l'État policier, fondé sur l'espionnage et les dénonciations ${ }^{29}$, comme le montre la première strophe du premier couplet d'Ultra :

« Ils ont régné sans limites,

Personne n'a bougé,

Car si quelqu'un avait osé,

Et dit une parole libre

(24) Ils apparaissent dans l'ordre inverse de la hiérarchie : d'abord le fonctionnaire municipal Klaus, puis le secrétaire particulier de la ville, le maire et enfin le prince Metternich.

(25) HKA 26/I, I, 7, p. 32 et III, 3, p. 57.

(26) I, 2, p. 10.

(27) II, 7, p. 45. L'expression est attribuée à Windischgrätz.

(28) «Zopf » désigne la tresse ou queue de cheval et renvoie à la perruque qui était à la mode pour les hommes au $\mathrm{XVIII}^{\mathrm{e}}$ siècle.

(29) Cette critique se retrouve dans Der alte Mann mit der jungen Frau II, 18. 
Il aurait été emprisonné,

On y était habitué.

Ils ont espionné tout le monde sans exception

C'était le rôle de la police.

Les gens intelligents se sont tus;

Bref, tout le monde était devenu stupide $[\ldots] »^{30}$.

Le gardien de nuit a été dénoncé comme libéral, le gouvernement sait qu'il lit la presse étrangère et Klaus le menace de la perte de son emploi s'il poursuit ses activités (I, 3). Le fonctionnaire municipal est « sur les traces d'une bande de libéraux » et a découvert qu'une auberge est « le foyer de la Révolution » $»^{31}$.

Cet État se caractérise par l'absence de droit et de liberté, comme l'explique Ultra à l'aide de jeux de mots dans le long commentaire qui suit son premier couplet : le régime a accordé à ses « sujet[s] opprimé[s] » « une quantité de droits » tels que les «droits de la naissance, les droits et privilèges de la condition sociale », le droit de prélever un impôt sur le vin, le droit de faire emprisonner quelqu'un pour insolvabilité etc., mais ils ne possédaient pas les droits humains fondamentaux : «Et malgré tous ces droits inestimables, nous n'avions aucun droit, parce que nous étions esclaves $\|^{32}$. De même, il existait toutes sortes de libertés : la liberté de commerce, la «liberté de masque » (Maskenfreiheit) qui supprimait les barrières entre les classes sociales dans les bals, la liberté de jouer au billard et même la liberté de pensée, à condition que les sujets gardent leurs pensées pour eux : «En un mot, nous avons eu une multitude de libertés, mais de la liberté aucune trace $»^{33}$. L'inflation de droits et de libertés restreints et futiles masque la négation du droit et de la liberté par un système d'oppression.

La censure est l'un des instruments de répression du régime, que dénonce avec virulence Nestroy ${ }^{34}$. Ultra la décrit comme « la plus jeune de deux sœurs abominables, l'aînée se nomme inquisition », elle est « l'aveu des grands [révélant] qu'ils ne savent qu'opprimer des esclaves abrutis, mais non gouverner des peuples libres », les Lumières l'ont marquée

(30) I, 7, p. 15-16.

(31) I, 6, p. 15. L'expression vient de Sedlnitzky.

(32) I, 7, p. 18.

(33) Ibid.

(34) Il en a été victime dès 1826. Cette critique revient dans les autres pièces, par exemple Der alte Mann mit der jungen Frau III, 3. 
« au fer rouge du mépris ». Le censeur est « un crayon devenu homme ou un homme devenu crayon, un trait devenu chair sur les productions de l'esprit $[\ldots] \gg{ }^{35}$. Les journalistes sont placés devant l'alternative suivante : écrire des banalités ou livrer une feuille blanche, tout article intéressant étant censuré (I, 8). Sont également critiqués le cléricalisme d'État et plus précisément les relations entre l'État et les Liguoriens, autre symbole de la réaction pour Nestroy: Klaus les considère comme le seul rempart contre l'épidémie de liberté qui se propage parmi le peuple (I, 16). Ils travaillent avec le maire à réprimer la liberté. Le Père prieur fait également cause commune avec lui dans l'affaire illégale du testament de l'époux de Madame de Frankenfrey (I, 13; I, 15). Au cours de scènes dénuées de satire, qui montrent l'hostilité de l'auteur, les habitants de Krähwinkel armés de hallebardes encerclent le monastère (III, 5-6) et en chassent les Liguoriens, tandis que le chœur célèbre la fin de l'oppression par les religieux (III, 12). Enfin, la bureaucratie, autre pilier du régime à côté de l'armée et de l'Église, est présentée comme hypertrophiée, cynique et corrompue (I, $11 ; \mathrm{I}, 14)$ ce qui accroît la dette publique (I, 12). Alors que leurs supérieurs perçoivent un salaire important pour « ne rien faire », les fonctionnaires subalternes sont pauvres et la peur de perdre leur emploi les contraint à un comportement servile (I, 12; I, 15).

\section{Les acquis de la Révolution : la liberté, l'égalité, le droit, la démocratie, l'émancipation du peuple et la défense de valeurs humanistes}

Ultra (dont le nom indique l'orientation politique : «ultraradicale »), qui prend la tête du soulèvement à Krähwinkel, oppose au pluriel des libertés et droits sous l'absolutisme, destiné à masquer leur absence, leur institution au singulier par la Révolution (I, 7). Ce qui paraît une « absurdité mathématique » se révèle une " vérité évidente »: seul le passage des libertés à la liberté et des droits au droit permet l'abolition des privilèges et l'instauration de l'égalité entre les citoyens. La pièce célèbre donc les acquis révolutionnaires, à commencer par l'égalité : «maintenant la naissance ne [vaut] plus rien $\gg^{36}$ déclare Ultra, ce que seules les sages-femmes peuvent regretter; et l'époque où le titre de "conseiller privé » était une distinction est révolue (III, 2). Du point de vue du droit, le protagoniste se réjouit de la suppression du Haslinger (I, 9-10). Et sur-

(35) I, 14, p. 27, 26.

(36) I, 7, p. 19. De même Die lieben Anverwandten III, 10 et Verwickelte Geschichte! I, 3. 
tout, il loue - et à travers lui Nestroy - la liberté qu'il incarne, comme le montre la scène où il décline son identité devant les fonctionnaires subalternes Sigmund Siegl et Willibald Wachs :

« Sigmund. Âge -

Ultra. Trois mois et demi. [...] Pas une heure de plus; c'est l'âge de la liberté, et ce qui précède ne compte pas.

$[\ldots]$

Sigmund. Nez -

Ultra. Humant la liberté.

$[\ldots]$

Sigmund. Taille-

Ultra. La moitié de la hauteur des barricades.

Sigmund. Signes particuliers -

Ultra. Esprit agité.

Sigmund. Caractère -

Ultra. Contraire aux règlements de police! $[\ldots] \aleph^{37}$.

Ultra ajoute - non sans ironie de la part de l'auteur - que les seuls à se plaindre de la liberté sont les poètes, privés du prétexte qu'ils invoquaient lorsque l'inspiration leur faisait défaut, la censure (I, 7). La pièce rend hommage aux journées du 13 mars, 15 mai et 26 mai 1848, les «trois beaux numéros » de la liberté (I, 22). La Révolution signifie en outre l'émancipation du sujet et l'instauration de la démocratie, comme le montre la troisième strophe du premier couplet d'Ultra :

«Alors le sujet opprimé

Se mit à réfléchir :

"Au diable,

Dois-je donc être esclave?

Le souverain est bien un seigneur,

Mais je suis homme comme lui;

Et même s'il nous en coûte la vie -

Nous demanderons maintenant

Pour tout des comptes

Aux puissants seigneurs" $[\ldots] \|^{38}$.

(37) I, 15, p. 29. Voir aussi : « Je suis la liberté même » I, 12, p. 24.

(38) I, 7, p. 16. 
L'importance et le rôle du peuple sont soulignés à plusieurs reprises (bien que Nestroy montre également le manque de sens politique de ce dernier, qui s'enthousiasme sans cesse) : Ultra critique le titre de « conseiller privé », en allemand « secret», en défendant l'idée qu' « un bon et honnête conseil ne doit plus maintenant être secret, le peuple entier doit pouvoir l'entendre $»^{39}$. Au maire qui demande qui ose chasser les Liguoriens, il répond : "Quelqu'un qui est des milliers de fois plus [puissant] que nous deux réunis, le peuple ${ }^{40}$ ! Ce dernier acquiert une conscience politique : le fourreur Schabenfellner déclare au maire, qui tente de s'opposer à l'expulsion des religieux : « Nous sommes un peuple libre $»^{41}$.

Plus généralement, les valeurs humanistes portées par la Révolution se retrouvent dans l'utilisation au sens propre, fort, du mot « Mensch », " être humain », par les révolutionnaires, tandis que les réactionnaires le réduisent à une interjection familière exprimant la surprise ou la crainte, voire à un terme péjoratif : "Vous êtes bien plus fourreur qu'homme $»^{42}$, reproche le gardien de nuit à Schabenfellner. Ultra répond à l'exclamation du maire, qu'il a percé à jour : "Si vous m'aviez donné un autre nom, j'aurais répondu : "Vous-même", mais puisqu'il en est ainsi - » et à la remarque méprisante de Reakzerl, qui s'étonne de sa présence dans le salon de Mme de Frankenfrey : «C'est vrai, le maire et un être humain fréquentent la même maison, c'est vraiment une société mélangée ! ${ }^{43}$. Tous ces acquis sont réunis - sur un mode parodique - dans la Constitution proclamée par Ultra déguisé en «commissaire européen de la liberté et de l'égalité » (II, 16).

\section{Le triomphe - provisoire? - de la Révolution et le spectre de la réaction}

La farce Freiheit in Krähwinkel est structurée autour de l'antithèse entre la réaction et la Révolution, l'ancien et le nouveau régime, le passé et le présent, au niveau de la dramaturgie (parties de l'action, personnages), des thèmes et de la langue (métaphores etc.). Elle n'est pas seulement une apologie de la Révolution et de la liberté, mais aussi une mise en garde devant le danger de la réaction. Le titre l'indique déjà par le paradoxe qu'il forme en rapprochant l'idéal politique et éthique de " liberté »

(39) III, 2, p. 56.

(40) III, 3, p. 58

(41) III, 6, p. 60.

(42) I, 1, p. 9.

(43) I, 14, p. 27 ; III, 2, p. 55. 
et «Krähwinkel », symbole de la petite ville étriquée. De même, la première partie (actes I et II) est intitulée «La Révolution », tandis que la seconde (acte III), "La réaction », semble annoncer le triomphe de cette dernière. Le fait qu'il en soit autrement à la fin de la pièce (mais non dans la réalité) s'explique davantage par les conventions du genre que par le dénouement logique des événements dramatiques.

Bien que ridicules, les personnages incarnant la réaction - associés aux métaphores de la nuit et de l'obscurité (I, 1; III, 14) - conservent un pouvoir important, dont se méfie Ultra : «Vous [Reakzerl] êtes réactionnaire et il ne faut jamais leur faire confiance $»^{44}$. Ils attendent, dans l'ombre, leur heure : "C'est aujourd'hui le jour de la vengeance, le triomphe de la réaction ${ }^{45}$, déclare le maire. Et Metternich (Ultra déguisé) affirme : «[...] la nuit aux étoiles étincelantes de la réaction triomphe[ra] dans le ciel politique $»^{46}$. En effet, tous, y compris Ultra, connaissent le fond réactionnaire des citoyens de Krähwinkel :

« Parmi les citoyens de Krähwinkel, beaucoup deviendront vraisemblablement mesquins et, après avoir brisé leurs chaînes, sans être véritablement réactionnaires, ils commenceront pourtant à se plaindre, pusillanimes : “Oh, mon Dieu, c'était mieux avant [...]" " ${ }^{47}$.

Klaus utilise le même argument pour rassurer le maire après ses cauchemars :

« Non, je connais les citoyens de Krähwinkel - il faut les laisser se défouler; une fois l'accès de folie passé, ils seront conciliants et nous les attraperons avec la main. Ensuite, nous serrerons bien fort [pour qu'il ne bouge plus], le peuple $»^{48}$.

Deux scènes (I, 21 et III, 3) annoncent la répression de l'insurrection par l'armée, même si celle de Krähwinkel est dérisoire (II, 2). En outre, le troisième rêve du maire (I, 25) et la rencontre entre ce dernier et Ultra déguisé en prince russe (II, 4-7) ont une fonction d'avertissement prophétique. La Russie tsariste était le principal allié des réactionnaires autrichiens. Elle apparaît comme plus despotique et tyrannique encore

(44) III, 2, p. 56.

(45) III, 3, p. 56.

(46) III, 14, p. 65.

(47) I, 7, p. 18-19.

(48) I, 24, p. 38. 
que le gouvernement autrichien du Vormärz (II, 4). Enfin, dans le couplet final d'Ultra (III, 22), deux strophes seulement sur cinq sont consacrées à la Révolution : la deuxième décrit la révolution en Irlande contre la suprématie anglaise et la troisième le soulèvement futur des Russes contre le tsar (elle s'oppose donc au troisième rêve du maire). En revanche, les strophes 1 et 4 représentent l'échec de la Révolution et la réaction : la première concerne la répression du soulèvement populaire au Royaume des Deux-Siciles, la troisième relate les événements qui se déroulent après la révolution parisienne (évoquée dans le premier couplet d'Ultra) : après l'abdication de Louis-Philippe, les révolutionnaires proclament la république et mettent en place un "gouvernement des prolétaires ». Mais de nombreux Parisiens « veulent de nouveau un roi » et « souhaitent un Napoléon $»^{49}$. Nestroy est ici visionnaire : après le coup d'État du 2 décembre 1851, Louis-Napoléon Bonaparte deviendra empereur sous le nom de Napoléon III en 1852. Ces événements montrent l'évolution dialectique de l'Histoire, qui instaure une nouvelle forme d'oppression.

La pièce s'achève certes par un dénouement heureux. Mais celui-ci est tellement exagéré - avec trois mariages et le triomphe de la Révolution - qu'il n'est pas crédible. En outre, l'union d'Ultra et de Mme de Frankenfrey est ambiguë : elle est le fruit de la Révolution, qui abolit les différences de classes, mais l'ascension sociale d'Ultra (dans le cercle des aristocrates et des capitalistes) peut aussi être interprétée comme une trahison des principes révolutionnaires ${ }^{50}$. On peut se demander si les jeunes filles déguisées en étudiants sont réellement un bastion contre la réaction et si la fuite du maire à Londres ne se justifie pas uniquement par la nécessité du dénouement heureux. Quant à la conclusion du protagoniste : « [...] la réaction est un fantôme, mais il est bien connu que les fantômes n'existent que pour les gens peureux; ne la craignons pas et il n'y aura pas de réaction! $»^{51}$, son caractère illusoire ne peut manquer de susciter le scepticisme.

Nestroy, tel une Cassandre ${ }^{52}$, met en garde ses concitoyens devant la fragilité des acquis révolutionnaires et la menace de la réaction. Celle-ci n'était pas seulement le rêve du maire dans la pièce, l'auteur voyait plus loin

(49) III, 22, p. 74.

(50) Cf. John R.P. McKenzie, « Political Satire in Nestroys's Freiheit in Krähwinkel », Modern Language Review 75/2, avril 1980, p. 332.

(51) III, 25, p. 77.

(52) Gerhard ScHMIEDER, Revolutionäre Entwicklung und, idealistische 'Satire : Studien zu Johann Nestroys 48er-Posse Freiheit in Krähwinkel, Thèse, Erlangen-Nürnberg, 1981, p. 52 (= 142). 
que le public qui, dans son enthousiasme, exigea qu'il supprime cette scène. Elle devint réalité en octobre 1848 avec le siège et la prise de Vienne.

\section{La réaction et ses conséquences (Lady und Schneider, Judith und Holofernes, Höllenangst, Der alte Mann mit der jungen Frau, 1849)}

Si Freiheit in Krähwnkel montre, non sans le mettre en question, le triomphe de la Révolution, Lady und Schneider, première farce de Nestroy après la répression brutale du soulèvement, présente un bilan désillusionné : la Révolution n'a pas lieu dans cette pièce, le tailleur Heugeign ne connaît pas d'émancipation politique et la conviction que le contentement est plus sage l'emporte à la fin sur le désir d'ascension sociale ${ }^{53}$. Les spectateurs furent déçus par la satire de la Révolution présente dans la pièce et Nestroy fut considéré par beaucoup comme un renégat politique. Dans la parodie Judith und Holofernes, l'auteur utilise le procédé traditionnel qui consiste à donner des traits viennois aux lieux et personnages de l'action: les allusions et lapsus (scènes 9, 14, 15) permettent de faire un parallèle entre la ville juive de Béthulie, assiégée par l'armée d'Holopherne, et le siège de Vienne par Windischgrätz (scènes 10, 14, 15, 21, 24). Le titre et le motif central d'Höllenangst (la superstition), farce écrite un an après la capitulation de Vienne, indiquent le retour de l'obscurantisme. La Révolution est un « rêve [...] [qui] a pris fin » (II, 17) et les ébauches du « monologue du destin » montrent le scepticisme de Nestroy quant à un changement des conditions sociales et politiques ${ }^{54}$. Enfin Der alte Mann mit der jungen Frau, où Kern, le porte-parole de Nestroy, qualifie la Révolution d' « ivresse de la liberté ${ }^{55}$, est une anticipation de l'avenir proche (l'action se déroule en 1850).

Les pièces montrent la restauration de l'ordre politique et social antérieur et l'aggravation des conditions économiques. Le calme est revenu et la réaction abolit les acquis de $1848^{56}$. Les instruments de la tyrannie et du

(53) On peut également observer un changement de perspective, les notions révolutionnaires évoluant du domaine public au domaine privé (par exemple I, 9).

(54) HKA 27/II Höllenangst, Jürgen HeIn (ed.), Wien, Deuticke, 1996, p. 177, 184.

(55) Ibid., / 1 Der alte Mann mit der jungen Frau, Urs Helmensdorfer (ed.), Wien, Deuticke, 1997, I, 7, p. 15. Dans Verwickelte Geschichte! Wachtl parle, lui, de « vertige ». HKA 29 Alles will den Prophet'n seh'n - Verwickelte Geschichte! Walter OBERMAIER (ed.), Wien, Deuticke, 1999, I, 9 , p. 122.

(56) Voir la métaphore des trois règnes de la nature dans le couplet I, 7 de Wendelin dans Höllenangst et les vers : « Si je pouvais, je renverserais / Toute l'année 1848. » I, 14, p. 29. 
pouvoir arbitraire, la violence militaire et l'espionnage, sont renforcés et la censure est rétablie. Judith und Holofernes critique la domination militaire de Windischgrätz, à travers le personnage caricatural d'Holopherne (scènes $1,7,9,24)$, ainsi que les dénonciations ${ }^{57}$ : le personnage de Daniel devient un dénonciateur perfide qui fait condamner par intérêt personnel et appât du gain des habitants de Béthulie (scènes 17-19). La hiérarchie sociale est réaffirmée ${ }^{58}$, le peuple de nouveau opprimé et dans une situation économique précaire (en témoigne la situation de Wendelin et de sa famille dans Höllenangst I, 7-8). La Révolution a entraîné une récession économique dont sont victimes en particulier les commerçants. Judith und Holofernes dépeint la pénurie alimentaire et le renchérissement du coût de la vie pendant le siège de Vienne, l'une des causes de la capitulation de la ville (scènes $10,11,15,16$ ).

Les anciens révolutionnaires ont été emprisonnés ou sont devenus des fugitifs, poursuivis par la justice. Dans Höllenangst, le baron von Reichthal est l'un de ces exilés, qui durent quitter l'Autriche par milliers (I, 14). Ce sujet est l'un des deux axes de l'action de Der alte Mann und der junge Frau : le "démagogue » Anton est arrêté, sa famille subit des interrogatoires humiliants (I, 2), le jeune homme parvient à s'échapper, mais doit se cacher dans un chalet d'alpage (acte III) et doit changer de métier (III, 2). Animé d'une profonde sympathie pour les idées révolutionnaires et ceux qui ont combattu pour elles, Kern fait preuve de bienveillance (I, 6), de respect (III, 2) et de compassion envers les victimes de la réaction, expliquant :

«Ce que vous [Anton] avez fait, des centaines de milliers de personnes l'ont fait, chacun ou presque l'a fait - que ce soit par l'action, le verbe ou la pensée. Qui peut dire, dans la crise que traverse l'Europe actuellement : « Je n'ai pas participé »-? La Révolution était dans l'air, chacun l'a inspirée et l'a donc aussi exhalée. Personne ne doit chercher à enjoliver les choses. L'un ou l'autre a davantage été remarqué, ce qui signifie, comme le dit Schiller : "Je choisis celui-ci parmi vous, mais vous avez tous pris part à sa faute." Aussi ayons la plus grande pitié de ceux qui sont touchés et remercions Dieu qu'ils ne nous aient pas choisis nous par hasard! $[\ldots] \gg^{59}$.

(57) Ce thème est également présent dans Der alte Mann mit der jungen Frau III, 2.

(58) Comme le montre le conflit entre les bourgeois parvenus et la noblesse de souche dans Der alte Mann mit der jungen Frau III, 4-10.

(59) HKA 27/I, I, 15, p. 23-24. 
Höllenangst et surtout Der alte Mann mit der jungen Frau critiquent l'arbitraire de la justice post-révolutionnaire et l'injustice des peines ${ }^{60}$. Dans la première, les petites gens sont arrêtés ou relâchés sans en comprendre les raisons, soumis à la volonté des puissants. Le protagoniste Wendelin souligne la différence de traitement des prisonniers selon leur rang (II, 10). Et la lettre de Reichthal au juge von Thurming révèle que les juges sont des instruments aux mains du gouvernement (II, 2). Der alte Mann mit der jungen Frau décrit les méthodes utilisées contre les suspects politiques :

« Kern. Vous revenez de la chasse?

Holler. Si on veut, nous avons fait plusieurs fois un peu de chasse à courre.

Kern. Qu'avez-vous chassé?

Holler. Quelques hommes.

Kern. Quoi -!?

Holler. Nous sommes une si petite principauté et avons un si grand nombre de criminels - rien que des criminels politiques, bien sûr $»^{61}$.

Cet échange est révélateur de l'atmosphère qui régnait en 1849 : les fugitifs politiques étaient traqués et le moindre soupçon suffisait à faire pendre un honnête citoyen. L'accusation de « participation à l'encouragement du crime de haute trahison » par l'huissier Schippl (I, 2) montre comment sont construites des preuves de culpabilité conduisant à une condamnation à mort. Quant au gardien Hartkopf, il est un exemple de la corruption des autorités (I, 12) ${ }^{62}$. La durée des peines, en particulier, est injuste, en ce qu'elle est plus importante pour les opposants politiques que pour les autres criminels : «Un voleur de mouchoirs en soie est condamné à trois mois de prison [...] Le criminel politique pour une brève ivresse de liberté à dix ou quinze ans $[\ldots] »^{63}$. De manière plus générale, Kern souligne l'arbitraire d'une justice qui punit certains et d'autres non, alors que l'esprit révolutionnaire s'est emparé de tous :

« Après la Révolution, il ne peut y avoir de peine juste. Selon la loi, des centaines de milliers de personnes méritent la mort - évidemment,

(60) De même Judith und Holofernes, scène 18.

(61) I, 6, p. 13.

(62) Karikaturen-Charivari mit Heiratszweck critique également les méthodes utilisées pour les procès en justice (III, 22, 24, 26, 27), ces passages furent censurés.

(63) I, 7, p. 15. 
cela ne va pas; alors l'un est fusillé, l'autre condamné à quinze ans de prison, celui-là à six semaines, un autre encore reçoit une médaille - et au fond, tous ont fait la même chose $»^{64}$.

Cependant, toute opposition politique a disparu dans cette pièce. Le seul espoir des fugitifs et prisonniers est l'amnistie ${ }^{65}$ (I, 7 et I, 14). Celle-ci est annoncée par un messager, tel un deus ex machina, dans la scène finale (IV, 30). Le dénouement heureux manque néanmoins : ceux qui se sont soustraits à leur peine doivent émigrer en Australie dans les huit jours qui suivent, ce qui est le cas d'Anton et de sa famille (IV, 31).

Les multiples facettes de la satire rendent difficile, voire impossible, une « récupération » politique de Nestroy par l'un ou l'autre camp. Le but de l'auteur était avant tout d'écrire des farces à succès ${ }^{66}$, ce qui n'exclut pas l'expression d'opinions politiques. Nestroy salue les acquis de la Révolution, en particulier la liberté (particulièrement la liberté d'expression et l'abolition de la censure) et l'expulsion des Liguoriens ${ }^{67}$. Mais il révèle les défauts des deux camps : de la Révolution comme de la réaction. Aussi n'est-il pas pertinent de lui reprocher un « changement de cap » après 1848. Les textes écrits pendant et après la période révolutionnaire ${ }^{68}$ montrent que l'auteur adopte une position sceptique, anti-idéologique, critiquant le comportement des révolutionnaires, mais en même temps excusant ces derniers condamnés à des peines souvent lourdes. La position politique de Nestroy n'apparaît que de manière indirecte et incomplète dans les pièces : l'auteur s'intéresse davantage aux aspects sociaux et humains que politiques au sens strict. Dans Der alte Mann mit der jungen Frau et Höllenangst, l'espoir d'un changement a disparu en période de réaction, mais restent les rêves et aspirations, et seules des initiatives individuelles peuvent atténuer les effets de l'oppression.

(64) I, 15, p. 24.

(65) Nestroy pensait sans doute qu'une amnistie générale serait proclamée en 1850, ce qui explique pourquoi il situe l'action de sa pièce en cette année. Elle ne le fut qu'en 1852.

(66) Ce qui explique que certains aspects de la réalité économique et sociale ne sont pas évoqués, cf. HKA 26/I, p. 100-102 et Wolfgang HäUsLER, Von der Massenarmut zur Arbeiterbewegung. Demokratie und soziale Frage in der Wiener Revolution von 1848, Wien, München, Jugend und Volk, 1979.

(67) Contrairement aux radicaux (cf. Fass dans Verwickelte Geschichte! I, 3 et I, 9), Nestroy est opposé au séparatisme (comme le montrent ses lettres et la strophe 5 du couplet d'Ultra III, 22). Il se distancie du communisme (Lady und Schneider II, 17; Verwickelte Geschichte! I, 3) et reste attaché à la notion de propriété (Freiheit in Krähwinkel III, 19).

(68) Cf. Gesammelte Briefe und Revolutionsdokumente (note 6). 


\section{Les raisons de l'échec de la Révolution}

«Le peuple est un géant dans son berceau, qui s'éveille, se lève, titube, écrase tout et finalement tombe plus bas encore qu'il ne l'était dans son berceau $»^{69}$.

L'échec de la Révolution s'explique par les faiblesses politiques du mouvement révolutionnaire, les défauts des Viennois en particulier et des hommes en général, enfin par la déformation des idéaux révolutionnaires dans la langue et la réalité.

\section{Les faiblesses politiques du mouvement révolutionnaire}

L'échec de la Révolution est d'abord dû à des raisons politiques : Nestroy montre que les révolutionnaires n'ont pas de véritable programme ni d'objectifs précis. Ils se révèlent incapables de planifier et de mettre en œuvre un changement réaliste des conditions politiques et sociales. Dans Die lieben Anverwandten, l'auteur critique l'absence de conscience politique et l'ignorance de nombreux électeurs des questions débattues à l'Assemblée nationale de Francfort :

« Beaucoup donnent leur voix et ne savent pas pourquoi;

Beaucoup se sont précipités pour aller voter pour Francfort

Qui ne connaissent rien de Francfort à part les saucisses $»^{70}$.

Nestroy déplore également le caractère superficiel des débats, qui portent souvent sur des questions de forme comme l'uniforme de la garde nationale $^{71}$, ou les querelles sur le mono ou le bicamérisme (suscitées par la Constitution Pillersdorf) qui oublient l'essentiel, l'importance d'une majorité libérale forte au Reichstag :

« Beaucoup s'exclament: Pas deux chambres! C'est en tout cas le pire! Mais une chambre sans unité, cela ne va pas non plus.

Le principal pour les chambres est la vue depuis la maison,

(69) HKA 26/II, Lady und Schneider, Judith und Holofernes, John R.P. McKenzIE (ed.), Wien, Deuticke, 1998, Lady und Schneider I, 8, p. 17.

(70) Ibid. 25/II, IV, 4, p. 75. Cette strophe est souvent évoquée pour expliquer le scandale suscité par la pièce. Sur la réalité historique des élections, cf. Günter BERGHAUs, op. cit., p. 211.

(71) Die lieben Anverwandten IV, 4 et Freiheit in Krähwinkel I, 1. 
Si elle donne derrière ou devant, à l'air libre [mot à mot: " vers la liberté $\gg] \gg\rangle^{72}$.

L'absence de programme précis de la Révolution est mise en évidence à la scène I, 10 de Freiheit in Krähwinkel ${ }^{73}$ : Ultra s'apprête à faire un discours qui déclenchera la Révolution, mais il est interrompu dès la première formule par les hourras et les applaudissements du peuple, qui n'a pas besoin d'en entendre davantage. Le protagoniste lui-même se caractérise par un manque de conscience politique : pour proclamer la constitution, il est revêtu d'un costume «fantaisiste, orné de rubans à sept couleurs et porte au lieu de plumes des drapeaux sur son chapeau, dans la main gauche un drapeau à sept couleurs $[\ldots] \gg{ }^{74}$. Nestroy dépasse l'analogie avec l'événement historique : son apôtre de la liberté est paré non des couleurs de la révolution, mais de celles plus poétiques de l'arcen-ciel; il tient un discours incohérent, qui se conclut ainsi : « pour éviter toute querelle sur ce point, il n'y aura pas de système du tout $\rangle^{75}$. Ultra se déguise en des personnages révolutionnaires (le commissaire européen, l'ouvrier) aussi bien que réactionnaires (le Prieur liguorien, le prince russe, Metternich) : les oppositions politiques ne sont pour lui que des masques, qu'il change à volonté.

Cette absence de conscience politique est une entrave à l'action efficace. Les citoyens de Krähwinkel ne font presque rien pour obtenir la liberté et leur modeste contribution a le plus souvent lieu hors scène. Lorsqu'ils brisent le Haslinger de Klaus, l'événement est rapporté et les spectateurs assistent uniquement au tumulte qu'il déclenche (I, 9-10). L'ironie est qu'il ne conduit pas à une action révolutionnaire ... mais au café voisin, où les habitants préparent un charivari pour le maire ${ }^{76}$. Pendant les combats de rue, ils sont soit spectateurs (II, 10-11 et 13), soit victimes (chœur des blessés II, 13). Après avoir perdu quelques dents, le gardien de nuit menace ses adversaires en ces termes : « Maintenant, on va parler durement $»^{77}$ ! Quant aux femmes, elles préfèrent que leurs

(72) HKA 25/II, Die lieben Anverwandten IV, 4, p. 74. Voir aussi la critique des débats interminables sur les droits fondamentaux au Reichstag dans Lady und Schneider I, 8 et Judith und Holofernes, scène 15.

(73) De même dans Lady und Schneider II, 25 et Verwickelte Geschichte! I, 3.

(74) HKA 26/I, II, 16, p. 52.

(75) Ibid. De même Fass réclame une «abolition générale [...] de tout ce qui existe » Verwickelte Geschichte! HKA 29, I, 3, p. 113.

(76) Pendant le Vormärz, les discussions politiques publiques ne pouvaient avoir lieu que dans les cafés et auberges.

(77) HKA 26/I, II, 13, p. 50. 
maris restent à la maison (II, 11). Les habitants de Krähwinkel ne jouent un rôle actif que dans l'expulsion des Liguoriens (III, 10-13). La Révolution réussit finalement non grâce aux mérites des révolutionnaires, mais à la ruse d'Ultra, qui dupe les réactionnaires. Le protagoniste se déguise successivement en Prieur liguorien : il fait avouer à Klaus que le maire a subtilisé la constitution de Krähwinkel (I, 17); en prince russe : il trompe le maire qui lui remet le document (II, 6); en « commissaire européen de la liberté et de l'égalité » : il proclame la liberté inscrite dans la constitution (II, 16); puis en Metternich, qui a fui Vienne, il parvient à retarder la répression militaire prévue par le maire (III, 14), afin de laisser aux révolutionnaires le temps d'ériger des barricades; enfin en ouvrier, il affirme que le principe "La propriété est sacrée » est gravé dans le cœur de ses camarades (III, 19), propos problématiques qui rassurent la bourgeoisie, mais privent les ouvriers de l'une de leurs revendications fondamentales.

Manque de conscience politique et incapacité à agir efficacement se traduisent dans la langue par la vacuité et l'absurdité du propos. La rhétorique révolutionnaire, l'emphase et le pathos de la liberté pallient l'absence d'un véritable programme, le vocabulaire libéral dégénère en phraséologie : Heugeign dans Lady und Schneider en est la caricature (par exemple II, 1). Nestroy critique avec virulence ce mauvais usage du langage - notamment par les poètes de la Révolution ${ }^{78}$-, en reprenant les mots d'ordre : « La victoire ou la mort! $»^{79}$ et les clichés, en particulier les métaphores du soleil, du printemps, de l'aube, du réveil associées à la Révolution : «Mon sombre destin me conduit de l'Autriche glorieuse, rayonnant de liberté, à Krähwinkel » explique Ultra lors de sa première entrée en scène; il qualifie le mépris du Haslinger de "premier rayon matinal du soleil de la liberté » et le charivari de « première hirondelle du printemps », ajoutant : « bientôt, la semence sera en pleine floraison $»^{80}$. L'élan révolutionnaire se réduit ainsi en grande partie à la rhétorique. La satire dénonce le décalage entre mots et actes, elle rend « visible la différence entre revendication verbale et accomplissement réel », selon Seeba. La pièce ne critique pas l'aspiration des citoyens à la liberté, elle

(78) Selon Hinrich C. SEeBA, « la Liberté à Krähwinkel [est] l'illusion présentée sous forme de farce des poètes de la liberté [à l'époque du Vormärz], qui prennent la parole pour l'action. » « Die Sprache der Freiheit in Krähwinkel. Anmerkungen zu Nestroys Revolutionssatire », dans Austriaca. Beiträg zur österreichischen Literatur. Festschrift für Heinz Politzer zum 65. Geburtstag, Winfried Kudszus et Hinrich C. Seeba (ed.), Tübingen, Niemeyer, 1975, p. 147.

(79) HKA 26/I, I, 10, p. 22, de même III, 24.

(80) I, 7, p. 17; I, 9, p. 21 ; I, 10, p. 23. 
ne met pas « [en cause] l'acte de la libération, mais la langue (qui le permet ou l'empêche) de la liberté »: « le caractère illusoire de la liberté à Krähwinkel » est dû au fait que « le mot se détache de la réalité », ce qui suscite l'« absence d'effet du mot vide $»^{81}$.

Une autre faiblesse du mouvement révolutionnaire est selon Nestroy la prise en compte insuffisante des difficultés économiques de la petite bourgeoisie :

« Le découpage qui sépare horizontalement l'humanité en « haut » et «bas », ne compte pas beaucoup dans la politique, évidemment, parce qu'à tout moment, le haut est en bas et le bas en haut. La politique ne se préoccupe que de la division verticale qui forme deux partis, la gauche et la droite $»^{82}$.

C'est l'une des raisons qui expliquent l'éclatement progressif (dès l'été 1848) du front révolutionnaire. En outre, la peur du communisme et le désir du retour à l'ordre inciteront la bourgeoisie à se rallier à la réaction. La première strophe du couplet IV, 4 de Die lieben Anverwandten, qui réunit les craintes de la population viennoise en mars-avril 1848, mentionne le déferlement d'un million de prolétaires franchissant le Rhin et l'effondrement des bourses européennes.

Enfin, les révolutionnaires méconnaissent le danger de la réaction. Les élections aux grades militaires (Chargenwahlen) ont permis à des réactionnaires d'intégrer les organes de pouvoir révolutionnaires, ils utilisent leurs postes afin de satisfaire leur vanité, voire se montrent ouvertement contre-révolutionnaires, comme l'explique le couplet IV, 4 de Die lieben Anverwandten :

«Peu sont animés d'un vrai sens révolutionnaire,

On voit, lorsqu'il s'agit d'une charge, combien ils aiment toujours commander;

Que ne feraient-ils pas, on n'en croit pas ses oreilles,

Ces incroyables manœuvres pour devenir sergent-chef! $»^{83}$.

(81) Hinrich C. SEEBA, op. cit., p. 138, 143.

(82) HKA 26/II, Lady und Schneider II, 13, p. 60. Voir aussi Die lieben Anverwandten: « Malheur à celui qui appartient aujourd'hui à la classe moyenne, car c'est lui qui est pillé. » III, 8, p. 61 et Judith und Hofofernes, scènes 10, 16.

(83) Ibid., 25/II, p. 75. 
Dans Freiheit in Krähwinkel, les propos de Klaus retentissent comme une mise en garde : « Aucun [espion] n'est encore mort de faim à cause de cela [la liberté], un signe qu'on les nourrit toujours secrètement $\rangle^{84}$. Les troubles favorisent le retour de la réaction: «Bagarrez-vous, portez la confusion à son comble, cela fait monter les actions de la réaction $»^{85}$ ! dit Reazerkl cynique. Le chaos est même entretenu par les réactionnaires afin d'atteindre leur but, comme l'explique Metternich (Ultra déguisé) : « La confusion générale que je nourris est l'obscurité crépusculaire, le flamboiement sanglant du soleil couchant, et la nuit aux étoiles étincelantes de la réaction triomphe[ra] dans le ciel politique. $\rangle^{86}$. Même après son exil, Metternich demeure au centre de la Contre-révolution, il prend congé du maire avec ces mots : "Si vous venez à Londres, rendez-moi visite. Tous les partisans serviles et légitim[ist]es de l'absolutisme viennent m'y rendre leurs devoirs $"\rangle^{87}$. Tout en approuvant la plupart des revendications des révolutionnaires, Nestroy est conscient des faiblesses politiques du mouvement et du pouvoir que conservent les réactionnaires.

\section{Les défauts des Viennois de l'époque et des hommes en général}

L'échec de la Révolution s'explique ensuite par les défauts des Viennois de l'époque, dont Nestroy doute de la maturité politique - et plus généralement de celle du peuple ${ }^{88}$-, défauts qui sont finalement ceux des hommes en général. Les Viennois ne prennent pas la Révolution suffisamment au sérieux, comme le répète le refrain du couplet IV, 4 de Die lieben Anverwandten : « Ma foi, pour cette époque sérieuse/ Il y a encore beaucoup de gens qui aiment plaisanter $»^{89}$. Les habitants de Krähwinkel, comme ceux de Béthulie ${ }^{90}$, conçoivent la Révolution comme un divertissement et un spectacle : le maître plombier Pemperl veut « voir un peu de révolution [...] Qui sait quand il y aura de nouveau une révolution? $\wedge^{91}$, les jeunes filles de Krähwinkel s'extasient devant les hommes en armes (III, 1) et regrettent l'absence d'étudiants (III, 4). Le peuple fête par des

(84) Ibid., 26/I, I, 3, p. 11-12.

(85) III, 3, p. 58.

(86) III, 14, p. 65.

(87) III, 15, p. 66.

(88) Voir la citation liminaire de la seconde partie.

(89) HKA 25/II, p. 73-76.

(90) Judith und Holofernes, scènes 16, 17.

(91) HKA 26/1, II, 11, p. 48-49. Voir aussi les scènes II, 10 et 13. 
" cris de liesse, bruits de trompettes et de timbales $\rangle^{92}$ la proclamation de la liberté. Le caractère théâtral, voire carnavalesque de la Révolution à Krähwinkel est souligné par les déguisements, costumes, gestes et réquisits : Ultra incarne, outre son propre rôle, cinq personnages différents, dont il emprunte les costumes à un directeur de troupe qui les a mis en gage (I, 15); pour celui du prince russe, il est accompagné de Willibald Wachs, qui lui sert d'interprète, et du gardien de nuit, qui joue un serf (II, 4); son arrivée à cheval en commissaire européen donne lieu à une scène théâtrale (II, 16-17); Willibald change de nouveau de costume pour construire les barricades (III, 17); les jeunes filles de Krähwinkel, sous la houlette de $\mathrm{M}^{\text {me }}$ de Frankenfrey, se déguisent en étudiants (III, 22-24). La Révolution à Krähwinkel est la répétition de celle qui a eu lieu à Vienne et auparavant en Allemagne et en France (I, 7) : au début du soulèvement, Ultra monte sur une chaise pour faire un discours à la manière d'Adolf Fischhof (I, 10), les révolutionnaires érigent des barricades en utilisant les pavés de la petite ville (III, 17), ils chantent l'hymne révolutionnaire, tandis que résonne une marche de Strauss fils (III, 23, 25).

Les (petits) bourgeois viennois sont ensuite décrits comme égoïstes et timorés. Beaucoup sont des suiveurs - «Il faut hurler avec les loups ${ }^{93}$ dit Wachtl (Verwickelte Geschichte! I, 9) -, qui pensent uniquement à leurs propres intérêts économiques, comme le fourreur Schabenfellner, prêt à soutenir la Révolution à condition qu'il puisse vendre aux gardes nationaux des bonnets de grenadiers (Freiheit in Krähwinkel I, 1 ${ }^{94}$, les habitants de Béthulie, qui spéculent sur les prix et suivent le cours de la bourse (Judith und Holofernes, scènes 10, 14), ou Fass, qui dresse une liste noire de ses futures victimes - les commerçants chez qui il a des dettes - si la Révolution triomphe (Verwickelte Geschichte! I, 4). Ils manquent de combativité - «Nos concitoyens / sont loin d'être bêtes / Ils n'ont aucun plaisir à combattre ${ }^{95}$ répète le refrain du couplet de Joab (Judith und Holfernes, scène 14) - et se démotivent rapidement, comme le montre la défection que connaît la garde nationale (scène 17) ${ }^{96}$. Une fois l'enthousiasme passé, les habitants regrettent le calme et l'ordre d'avant la Révolution (Freiheit in

(92) II, 16, p. 52.

(93) HKA 29, p. 121.

(94) Dans les trois premières scènes de Freiheit in Krähwinkel, la discussion sur la liberté est rapportée par des jeux de mots à la profession des habitants.

(95) HKA 26/II, p. 95. Voir aussi les scènes 9, 11, 16, 17.

(96) Voir aussi les doutes d’Ultra : «Krähwinkel n’est pas Vienne, ni Paris, ni Berlin. Auront-ils ici la ténacité nécessaire? », HKA 26/I, III, 4, p. 57. 
Krähwinkel I, 7 et I, 24). Lorsque la situation se retourne, ils prennent peur et subissent sans broncher la réaction ${ }^{97}$.

Ce caractère explique leur versatilité et la soumission servile à celui qui les domine. La satire de Nestroy vise tout particulièrement les opportunistes, type incarné par Heugeign dans Lady und Schneider, «l'homme » qui veut être "à la tête » d'un mouvement, selon le titre originel (Der Mann an der Spitze) : "Il faut qu'ils me placent à la tête de quelque chose, d'un mouvement ou d'un club, libéral, légitimiste, conservateur, radical, oligarchique, anarchique ou pas anarchique, cela m'est égal, juste à la tête $\rangle^{98}$ ! Le comportement d'Heugeign, comme celui de Fass dans Verwickelte Geschichte! (I, 4), révèle une certaine mégalomanie, voire folie ${ }^{99}$. L'auteur dénonce plus généralement les «girouettes » qui varient au gré du vent :

« Seul un commerce n'a pas son compte

Qui, au sens métaphorique,

A le plus à faire,

Ce sont les selliers, qui s'activent

Énormément en cette époque,

Où du jour au lendemain,

Des centaines de milliers de gens

Ont si intelligemment changé de selle

Et pas un parmi eux

Ne paie les selliers. [...] $»^{100}$.

De même dans la cinquième strophe du couplet I, 14 de Wendelin dans Höllenangst ${ }^{101}$. Les poètes ne sont pas épargnés comme le montre Sperling Edler von Spatz dans Freiheit in Krähwinkel, qui écrit « Ode au Bundestag », puis à l'occasion de la visite du prince russe « Impromptu au knout » (II, 3) et enfin « À la liberté » (III, 1). L'entreprise révolutionnaire est vouée à l'échec parce que l'engagement politique de l'homme est motivé par des raisons égoïstes. Les défauts des hommes font qu'ils ne sont pas à la hauteur des idéaux de la Révolution.

(97) Voir la strophe 2 du couplet II, 6 de Wachtl dans Verwickelte Geschichte!

(98) HKA 26/II, I, 10, p. 23. De même I, 34.

(99) I, 10, 11, 26; II, 9, 29.

(100) I, 8, p. 16.

(101) Voir aussi Der alte Mann mit der jungen Frau I, 5. 


\section{La déformation des idéaux dans la langue et la réalité}

«Ô liberté et égalité! Ce que tu vas devenir est une véritable Passion! ${ }^{102}$

Si l'on considère à un troisième niveau (après la petite ville étriquée et l'image de Vienne) Krähwinkel comme la représentation de l'humanité imparfaite, la question posée par Nestroy dans la farce est alors : qu'advient-il de la notion de liberté quand elle est instaurée dans la réalité? L'instauration de la liberté à Krähwinkel vide la notion de son sens - ici se manifeste le scepticisme de Nestroy par rapport à la tradition idéaliste. Le terme prend des significations multiples et parfois contradictoires : à la scène I, 1, le chœur des habitants revendique dans une formule paradoxale la liberté, non parce qu'elle est pour eux un besoin vital, mais pour faire comme les autres capitales européennes :

« Le monde entier savoure la liberté,

Nous seuls, habitants de Krähwinkel, ne l'avons pas.

Les citoyens de Krähwinkel, sapristi,

Sont aussi un élément allemand.

Aussi, nous ne céderons jamais, il nous faut la liberté!

Nous l'obtiendrons, même s'ils nous emprisonnent à perpétuité $»^{103}$.

La liberté devient un mot d'ordre, qui perd son sens concret et universel. À la scène suivante, le gardien de nuit dit à Klaus : "Nous prendrons cette liberté ${ }^{104}$, expression que le fonctionnaire municipal interprète comme un programme politique et à laquelle il répond : "Nous n'aimons pas que l'homme soit libre. » Les femmes de Krähwinkel, quant à elles, s'opposent à la liberté qui donne à leurs maris l'occasion d'aventures extraconjugales (III, 1). Enfin, les poètes de circonstance, qui se multiplient au cours de l'année $1848^{105}$, en banalisent le sens, comme le montre le poème de Sperling "À la liberté », prétexte à des rimes en «-ei » (III, 1). La scène III, 1 est un exemple des glissements sémantiques que subit la notion, chacun lui donnant une signification différente ${ }^{106}$.

(102) HKA 25/II, Die lieben Anverwandten III, 15, p. 68.

(103) Ibid., 26/I, I, 1, p. 5.

(104) I, 2, p. 10, allusion à la pointe finale du poème d'Anastasius GRÜN « Scène de salon » : «Aurais-je la liberté d'être libre? », Hambourg, 1831.

(105) Cf. Günter Berghaus, op. cit., p. 230.

(106) De même Verwickelte Geschichte! I, 9. 
Dans Lady und Schneider, Nestroy pousse cette logique à son terme dans le contexte post-révolutionnaire. Il montre que les hommes par bêtise ou à cause d'un défaut fondamental - transforment les idéaux en contraire de leur signification originelle :

« Ils croient que la liberté signifie râler librement contre l'État

Et prendre ce dont on a besoin à celui qui l'a. [...]

Ah, quand la liberté devient le communisme,

Cela cesse d'être un plaisir $»^{107}$.

Nestroy oppose par la parodie et la satire ${ }^{108}$ la grandeur des idées révolutionnaires à la médiocrité de leurs défenseurs, qui en détournent le sens pour en faire des mots d'ordre ou des formules creuses.

Au-delà de ce décalage imputable aux faiblesses des hommes, Nestroy montre que les idéaux ne sont pas compatibles avec la réalité : «[...] beaucoup de belles idées, lorsqu'elles entrent dans la vie, tournent mal ${ }^{109}$. regrette Wendelin dans Höllenangst. Le titre « Liberté à Krähwinkel » soulève déjà le problème de la réalisation de l'idéal de liberté : «à » signifie une altération de l'idéal. Le paradoxe met en évidence les défauts des citoyens de Krähwinkel (et des Viennois, voire de l'humanité), mais Nestroy se demande également si l'idéal de liberté peut se concrétiser dans la réalité. Cette question rejoint la contradiction inéluctable entre idéal et réalité, mais en les mettant tous deux en cause, ce qui explique la satire des idéaux révolutionnaires (liberté, égalité) présente dans certaines scènes (II, 15-16).

La réponse est clairement négative dans Lady und Schneider et Verwickelte Geschichte! où est fait un mauvais usage des libertés : de la liberté de la presse (qui permet d'écrire tout et n'importe quoi ${ }^{110}$ ), de réunion et d'expression (dont abusent les opportunistes et qui favorise les discussions de café du Commerce $\left.{ }^{111}\right)$. Le pathos de la liberté se prolonge et se concrétise dans des fêtes de la fraternité, des beuveries et des

(107) HKA 26/II, II, 17, p. 67.

(108) Selon la définition qu'en donne Friedrich SCHILLER dans De la poésie naïve et sentimentale: «Dans la satire, la réalité est opposée comme défaillante à l'idéal qui est réalité suprême ». Cité d'après Georgina Baum, Humor und Satire in der bürgerlichen Ästhetik. Zur Kritik ihres apologetischen Charakters, Berlin, 1959, p. 163.

(109) HKA 27/II, I, 7, p. 16.

(110) Lady und Schneider I, 8.

(111) Ibid., I, 10; Verwickelte Geschichte! I, 3. 
jeux militaires ${ }^{112}$ : la liberté devient licence. Nestroy montre de même la conception erronée qu'ont de l'égalité (sociale) ses défenseurs les plus extrêmes, pour qui « il ne doit exister aucune différence [de rang et de condition] entre un cordonnier et un duc »: les inégalités de fortune entre riches et pauvres sont d'autant plus flagrantes et il est moins aisé de traiter avec un petit bourgeois parvenu qu'avec dix nobles ${ }^{113}$. Les idées révolutionnaires se déforment inévitablement entre les mains des petits bourgeois du type de Heugeign et Fass ${ }^{114}$. L'incompatibilité entre idéal et réalité est problématisée dans le couplet politique d'Heugeign (II, 17), qui oppose l'agréable et l'utile : les cinq strophes montrent que «le peu de choses agréables» dans la vie humaine correspond rarement à l'utile, «mais malheureusement se transforme [...] presque toujours en quelque chose de désagréable $\gg^{115}$. Le couplet est construit autour d'oppositions (par exemple entre la ménagère et l' " amazone » sur les barricades dans la première strophe) et chaque strophe montre l'évolution d'une idée, qui va de la confirmation à la destruction de la belle image qu'on peut s'en faire : la première partie décrit l'idéal, une belle représentation (dans la première strophe : une femme intelligente, dans la deuxième : un appartement agréable, dans la cinquième : la vie à Vienne) ou une notion (dans la troisième strophe : l'égalité, dans la quatrième : la liberté); la deuxième montre qu'il n'est pas réalisable dans le contexte de l'époque. « Mais nos belles idées ne veulent malheureusement pas se réaliser ${ }^{116}$, déplore Fass (Verwickelte Geschichte! I, 3). Aussi belle que puisse être l'idée de liberté et d'égalité, l'homme ne peut rien en tirer, parce que les idées, dès qu'elles entrent dans la réalité, sont perverties, comme le montrent les deux vers du refrain cité plus haut : «Ah, lorsque la liberté devient le communisme, non, / Cela cesse d'être un plaisir. » La satire est à peine atténuée par le caractère fictif des exemples, la récurrence de l'expression «vu sous cet angle » (qui laisse la possibilité d'envisager les choses autrement, de manière plus positive) et par le fait que les strophes 3 et 4, dans lesquelles la satire est la plus féroce, sont précédées et suivies par des strophes traitant de sujets plus « légers », moins politiques.

(112) Freiheit in Krähwinkel III, 1; Lady und Schneider II, 1; Judith und Holofernes, scène 11 ; Verwickelte Geschichte! I, 3.

(113) HKA 26/II, II, 17, p. 66-67. Cette critique des parvenus a suscité des malentendus et conduit certains à taxer l'auteur de réactionnaire.

(114) Ainsi la fraternité et l'unité dans Verwickelte Geschichte! I, 3.

(115) Ibid., p. 64.

(116) Ibid., 29, p. 113. 
«C'était un penseur, et il ne pouvait donc être ni libéral, ni antilibéral ${ }^{117}$. L'observation et l'analyse inlassables de la réalité permettent à Nestroy de parvenir à une appréciation lucide et nuancée de la Révolution de 1848. Les comédies politiques sont le miroir de ce processus. Nestroy n'est ni l'acteur qui vient en costume de théâtre de la garde nationale sur les barricades (comme le représente une aquarelle de l'époque ${ }^{118}$ ), ni l'opportuniste qui exploite dans ses farces les événements sanglants (comme le prétendent certains critiques), mais un auteur dramatique qui, par la satire, s'efforce de donner une image la plus proche possible de la réalité de l'époque : les comédies politiques sont les composantes d'un tableau d'ensemble qui, à la fois, suit l'évolution historique et montre, en l'éclairant sous des angles différents, que le monde ne change pas. Bien que défendant les principes de la Révolution, Nestroy porte dès le début un regard clairvoyant sur les faiblesses du mouvement et le caractère humain, limité dans la pensée comme l'action, de ses partisans. Aussi critique-t-il aussi bien l'extrémisme de $"$ gauche ${ }^{119}$ que le conservatisme de $《$ droite $»^{120}$. La position de Nestroy est finalement celle de l'auteur satirique qui révèle les contradictions des deux partis, de la Révolution comme de la réaction, et qui, sceptique, voire pessimiste, ne croit guère en la possibilité d'une amélioration des conditions politiques et sociales - en tout cas dans un futur proche, comme le montre la conclusion de Verwickelte Geschichte! « Ô Allemagne, tu es encore loin du but » ${ }^{121}$. Du point de vue dramatique, les comédies politiques posent la question de la satire, à une époque qui lui est hostile (à l'exception des quelques mois au cours desquels la censure fut abolie, mais restait la pression exercée par le public). L'auteur recourt au monde en apparence harmonieux de la farce, mais transforme la signification des motifs et formes comiques traditionnelles : il interprète son «modèle » comme la réalité politique et sociale à critiquer par la satire. En alliant genre de la farce ou de la parodie et critique de l'époque - procédé qui ne réduit en rien la portée et l'effet de la satire, au contraire -, en modifiant la fonction des éléments traditionnels,

(117) Karl KraUs, op. cit., p. 25.

(118) SW V, p. 592.

(119) Par exemple dans Lady und Schneider : « il serait la plupart du temps souhaitable que la gauche soit plus modérée », HKA 26/II, II, 13, p. 60.

(120) « Oui, c'est l'erreur principale de la gauche comme de la droite : rien n'avance. », ibid.

(121) HKA 29, p. 146. Voir aussi deux vers d'une ébauche de monologue pour Höllenangst: « Puisque nous avons rêvé de liberté, elle doit réellement exister,/ Même s'il faut des siècles pour l'instaurer. », HKA27/II, p. 199. 
Nestroy dépasse les conventions de la farce et de la parodie viennoises pour se rapprocher - en particulier dans Freiheit in Krähwinkel -, de la " comédie politique » ou d'un «nouveau Volksstück» au sens de Brecht, à la fois artistique et proche de la réalité, animé d'une intention didactique et critique ${ }^{122}$.

Fanny Platelle

ANR THEREPSICORE

Centre de Recherches sur les littératures et la socio-poétique (CELIS)

Université Blaise-Pascal

Maison des Sciences de l'Homme

4 , rue Ledru

63000 Clermont-Ferrand

www.univ-bpclermont.fr/celis/

(122) Selon Bertold BRECHT, le Zeitstück doit « dépasse[r] la représentation de la situation [...] pour critiquer ou discuter la situation générale, une fois cette situation connue », Über Politik auf dem Theater, Frankfurt a. M., ${ }^{2} 1973$ [édition suhrkamp 465], p. 12. Judith und Holofernes a influencé L'irrésistible ascension d'Arturo Ui (1947). 